\title{
Filigrane
}

Écoutes psychanalytiques

\section{Premières consultations et symbolisation primaire}

\section{Vincent Cornalba}

Volume 27, numéro 2, 2018

La consultation psychanalytique aujourd'hui, entre héritages et remaniements

URI : https://id.erudit.org/iderudit/1055754ar

DOI : https://doi.org/10.7202/1055754ar

Aller au sommaire du numéro

\section{Éditeur(s)}

Revue Santé mentale au Québec

ISSN

1192-1412 (imprimé)

1911-4656 (numérique)

Découvrir la revue

Citer cet article

Cornalba, V. (2018). Premières consultations et symbolisation primaire.

Filigrane, 27(2), 113-124. https://doi.org/10.7202/1055754ar

\section{Résumé de l'article}

L'auteur souligne l'importance des conditions à respecter pour l'émergence d'un travail de symbolisation. La question de la symbolisation primaire et des carences qui ont pu l'accompagner dans les premières rencontres avec l'objet devient le socle à partir duquel les questions du cadre et du transfert sont évoquées. Le registre de la matrice de contact et la fonction du répondant sont alors tout particulièrement étudiés, en tant qu'ils représentent des outils précieux pour prendre en compte l'impact du rapport à la temporalité au décours de la dynamique psychothérapique : l'émergence d'un travail de symbolisation ne peut se réaliser sans la confrontation de rythmes que le fait institutionnel est en mesure de contenir, fonction complémentaire à la dynamique d'appropriation subjective engendrée par la situation analytique.
Ce document est protégé par la loi sur le droit d'auteur. L'utilisation des services d’Érudit (y compris la reproduction) est assujettie à sa politique d'utilisation que vous pouvez consulter en ligne.

https://apropos.erudit.org/fr/usagers/politique-dutilisation/ 


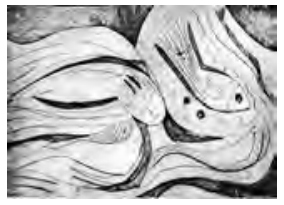

\title{
Premières consultations et symbolisation primaire
}

\section{Vincent Cornalba}

Résumé: L'auteur souligne l'importance des conditions à respecter pour l'émergence d'un travail de symbolisation. La question de la symbolisation primaire et des carences qui ont pu l'accompagner dans les premières rencontres avec l'objet devient le socle à partir duquel les questions du cadre et du transfert sont évoquées. Le registre de la matrice de contact et la fonction du répondant sont alors tout particulièrement étudiés, en tant qu'ils représentent des outils précieux pour prendre en compte l'impact du rapport à la temporalité au décours de la dynamique psychothérapique: l'émergence d'un travail de symbolisation ne peut se réaliser sans la confrontation de rythmes que le fait institutionnel est en mesure de contenir, fonction complémentaire à la dynamique d'appropriation subjective engendrée par la situation analytique.

Mots clés: symbolisation primaire; troubles narcissiques et identitaires; fonction du répondant; matrice de contact.

\begin{abstract}
The author emphasizes the importance of the conditions to be respected for the emergence of symbolization work. The question of primary symbolization and deficiencies which may have accompanied it in the initial encounters with the object becomes the basis upon which the questions of setting and transference are brought up. The dimension of the contact matrix and the function of the respondent are then most particularly studied insofar as they represent invaluable tools for taking into account the impact of the relationship of temporality to the waning of the psychotherapeutic dynamic. The emergence of symbolization work cannot occur without comparing the rhythms that the institutional reality is in a position to contain, a function complementary to the dynamic of subjective appropriation engendered by the analytic situation.
\end{abstract}

Keywords: primary symbolization; narcissistic and identity disorders; function of the respondent; contact matrix.

[...] la névrose apparaît généralement comme une sorte de «jeune fille venue de loin». $\mathrm{Ne}$ sachant pas d'où elle vient, on s'attend à la voir un jour disparaître. (Freud, 1913, p. 87).

ette citation de Freud peut nous servir de véritable fil rouge dans le cadre de cette contribution. Encore nous faut-il obvier, d'entrée, à tout malentendu. Il ne s'agira pas de commenter un quelconque diagnostic 
différentiel, comme pourrait le suggérer cet emprunt à l'œuvre freudienne. Cette approche serait bien trop éloignée du mouvement par lequel il convient d'appréhender les enjeux multiples d'une rencontre aux vertus psychothérapiques. Au centre de cet écheveau, il faut faire une place particulière au champ de la symbolisation, aux conditions qui en permettent l'émergence. Le travail analytique s'appuie d'ordinaire sur une métapsychologie de l'absence. Une absence que l'on envisage comme symbolisable et qui promouvrait une mise en jeu élaborative, à travers un circuit de représentations partageables. Mais qu'en est-il de ce travail psychique spécifique lorsque les fondements mêmes de cette symbolisation n’ont pas été assurés, lorsque ce qui aurait dû advenir pour le sujet dans les premières rencontres avec l'autre n'a pas eu lieu?

L'ensemble des contributions lors du Colloque qui s'est tenu à Montréal le 29 octobre 2016 témoignent d'une vision de l'apport psychanalytique en institution. Un apport qui semble s'établir à partir de la prise en compte des impasses de la symbolisation auxquelles grand nombre de thérapeutes sont aujourd'hui confrontés. Comment promouvoir un travail d'élaboration psychique lorsque le symptôme exprimé ne peut pas encore intégrer le champ du symbole pour le patient lui-même? Car il ne sert à rien que le clinicien en appréhende le sens si le patient, pour sa part, ne peut l'intégrer dans un circuit d'inscriptions qui promeut le registre de l'appropriation subjective. Dès lors, une certaine clinique, que d'aucuns ont coutume de référer aux souffrances narcissiques-identitaires (Roussillon, 2013), renvoie invariablement aux conditions premières pour qu'une symbolisation émerge et que les enjeux qu'elle recèle puissent être enfin partagés «en présence de ${ }^{1}$.

Une situation qui m'apparait d'autant plus riche qu'elle interroge donc les conditions mêmes d'une possible rencontre avec l'autre, en tant que toute rencontre serait à considérer comme l'élément déterminant qui initierait un effet de symbolisation à l'égard d'un événement passé, vécu initialement par un sujet seul, décidément trop seul. Ces questions ont depuis longtemps été débattues, mais leur reprise relève toujours autant de l'essentiel. À plus forte raison lorsqu'elles entrent en résonance avec d'autres questions traversées, débattues, mais aussi refoulées, répudiées, au sein de la structure dans laquelle elles prennent forme. J'ai toujours été sensible à cette capacité chez certains patients - une aptitude formée à leur insu - d'interroger aux premiers temps de la rencontre des registres laissés en suspens par l'institution accueillante et qui accèdent dès lors à une reviviscence potentiellement féconde. 


\section{Aux sources de la symbolisation}

Certains patients, reçus dans nos cabinets ou au sein de nos institutions respectives, nous (re) viennent de loin. Et si l'on continue de filer la métaphore freudienne, le risque est parfois grand de les voir arrêter leur cure en disparaissant, purement et simplement, sans autre forme de procès. Je le répète ici: si j'ai convoqué en exergue une certaine citation de Freud, ce n'est pas tant pour introduire la question du diagnostic différentiel que pour insister sur le registre de la méconnaissance, à l'origine de toute démarche vers un travail analytique. Car c'est bien de méconnaissance dont il s'agit et qui détermine la venue auprès d'un psychanalyste. Une méconnaissance dont V. Jankélévitch précisait en ces termes l'influence sur l'appréhension que l'on acquiert de soi et du monde:

Le problème de la méconnaissance concerne le mystère impalpable de la personne, de l'œuvre ou du message: la méconnaissance est une façon particulièrement irritante, controversable et aporétique de manquer celui-là [le je-ne-sais-quoi] en croyant connaître ceux-ci [la personne, l'œuvre, le message]. (Jankélévitch, 1980, p. 13).

Un «je-ne-sais-quoi», qui apparaît à la fois anodin et essentiel, et dont les plus petites occurrences échappent à la saisie. On est en droit, bien entendu, de convoquer l'expression de l'inconscient et des outils dévolus à l'appareil psychique afin de restituer mais aussi de réduire l'impact que cette relation d'inconnu produit sur le sujet. On le sait depuis Freud: l'étranger est agissant chez chacun et se manifeste sur un mode potentiellement inquiétant. Le travail de symbolisation pour lequel le psychisme humain est formé s'appuie sur la constitution de représentations partageables que les mythes et les croyances répertorient au sein de toute culture et dont la mission consiste à contenir l'angoisse que cette méconnaissance est toujours susceptible de produire chez l'être humain. On peut être tenté d'interroger ce fonds commun de représentations sur la souffrance psychique pour tenter d'expliquer les tenants de cette méconnaissance inaugurale chez tout patient: qu'en comprend-il? Quelle «logique» est associée au registre de la souffrance psychique? Dans quel modèle, dans quel schème de pensée, s'inscrit-elle pour le sujet singulier? La dimension culturelle est peut-être alors à convoquer. Mais je ne peux me soustraire à l'idée qu'il y a là quelque chose de bien plus universel, dès lors qu'un sujet n'a pas pu rencontrer ce proche capable de transformer des quantités d'excitations en éléments psychiques, 
de les qualifier affectivement et de les rendre accessibles à la représentation. Pour le dire autrement, tout sujet peut se vivre étranger à lui-même, non du fait de quelque particularité culturelle en inadéquation avec la société hôte, mais à cause d'une absence inaugurale, celle d'une vicariance psychique indispensable, en réponse à l'impuissance de l'infans pour qualifier certaines expériences rencontrées. Et cela renvoie à un lien d'évidence:

Winnicott relie le vide pathologique à un défaut du regard de la mère, ou pour le dire autrement à un défaut de la fonction subjectivante de l'environnement primaire. Il propose une formule extrêmement éclairante pour caractériser ce processus: là où il aurait dû se produire quelque chose, rien ne s'est produit. (Pinel, 2011, p. 10-11).

On est ici renvoyés aux conditions inaugurales permettant l'instauration d'une œuvre de symbolisation et l'avènement de ce qui va qualifier l'expérience en tant qu'elle pourra être investie, pensée, utilisée. C'est là la notion de Nebenmensch (Freud, 1895) qui s'affirme. Et cette fonction n'est-elle pas ce qui constitue le noyau même d'un certain accompagnement clinique, lorsque le clinicien fournit au patient, dans l'actuel de la rencontre, ce qui aurait dû être la réponse historique de l'objet, pour paraphraser A. Green? Une fonction qui se définit à partir de cette capacité à accueillir «les formes polymorphiques d'associativité» (Roussillon, 2013) jalonnant, ponctuant, le travail psychothérapique en présence d'un autre. Pour ce faire, il faut donc l'utilisation nécessaire d'un autre, mais d'un autre d'une certaine trempe - le Nebenmensch, c'est-à-dire ce sujet-autre proche et secourable à la fois, en constitue le modèle - dont l'aptitude à ressentir l'état de désaide précipite les conditions d'une métabolisation, avec à la clef une possible formulation de ces éléments dans un circuit de pensée enfin partageable. Pour ma part, c'est bien de cette symbolisation primaire - et des conditions de sa transmissibilité - dont il est principalement question: nous sommes rendus témoins d'une expérience princeps, celle qui permet l'établissement d'un échange de pensée avec l'autre pour que le sujet puisse enfin se re-présenter certains événements non subjectivés.

Événements traversés sous le régime du traumatisme. Insistons néanmoins sur le fait que la reconnaissance de ce traumatisme ne peut s'établir qu'à travers la qualité de présence d'un autre, en sa fonction de traducteur mais aussi de surface d'impression de la charge qui s'est exercée, par le passé, sur le sujet lui-même. Et il est loisible de s'appuyer sur la définition 
winnicottienne de la crainte de l'effondrement, un effondrement psychique qui a déjà eu lieu mais qui n’a pu être vécu par le sujet, car non éprouvé (Winnicott, 1975), pour appréhender l'enjeu principal de la dynamique psychothérapique dans ce type de situation clinique: le sujet devient dès lors tributaire de la capacité rencontrée chez un autre d'accueillir mais aussi de supporter et de transformer pour lui-même l'impact de l'effraction réalisée. Dans cette clinique particulière, il est fondamental de ne point convoquer d'emblée un quelconque canevas interprétatif susceptible d'écraser l'expérience à subjectiver par du déjà pensé. Car on mesure combien toute préconception théorique pourrait noyer ce qui demande à être pensé pour la première fois. Pensé, et dans le même temps, partagé au moment même où une certaine compréhension du fait qui fut traversé mais non vécu se fait jour. En fait, ces deux occurrences - penser et partager - sont, dans ce temps précis de l'analyse, synonymes. C'est au travers d'adresses en deçà du langage parlé qu'une certaine dynamique peut enfin s'enclencher: «Les formes de la symbolisation primaire concernent des expériences subjectives "précédant l'apparition du langage verbal" selon la formule de Freud de Construction en analyse.» (Roussillon, 2013, p. 42) C'est en effet à partir des résidus sensoriels et des pré-formes psychiques qui leur sont associées que le travail d'appropriation subjective devient possible... mais - insistons-le au risque de la répétition - à la condition qu'un autre, médiateur en présence, en accuse réception. C'est certainement ce qui se passe lorsque certains patients, en séance, «boivent» littéralement les paroles par lesquelles son thérapeute exprime ce que ça lui fait ${ }^{2}$. On est là dans ces moments où le travail de représentation ne peut se réaliser qu'en se reliant à une expérience sensorielle dans la rencontre avec l'autre et par un autre qui peut enfin en dire quelque chose. L'amorce du travail de symbolisation est assurée par un certain parcours à établir dans le cadre de la rencontre psychothérapique: un circuit qui passe par l'autre, qui le traverse et qui entraîne une qualification, jusqu'à présent refusée, à l'expérience. Fort de cette analyse, certains cliniciens ont développé des aménagements dans la pratique analytique, plus précisément au sein de l'approche groupaliste. On peut, à titre d'exemple, évoquer l'usage qu'O. Avron faisait à l'occasion de la figure du double, dans des moments d'impasse où le sujet, bien qu'à l'orée d'un mouvement d'appropriation subjective, se figeait dans un suspens. Le redémarrage du processus de symbolisation nécessiterait alors la part active du thérapeute: il ne s'agit pas de dire ce que le sujet n'est pas encore prêt à entendre, mais plutôt de poser des mots sur ce qui s'est déjà formulé à travers d'autres canaux de 
communication. L'expression du corps devient le viatique de cette tentative de formulation:

Je viens alors me placer près du sujet et j'exprime à haute voix en son nom ce que le patient, pour des raisons diverses que j'examinerai, ne peut encore traduire par sa propre voix mais qui est cependant suffisamment proche de son conscient. Cette technique utilisée avec parcimonie et à bon escient peut renforcer le contact du sujet avec ses propres éprouvés et provoquer par là même une dynamisation associative, en particulier chez des patients présentant des problématiques identitaires. (Avron, 2010, p. 96)

Certes, cette technique ne va pas sans susciter des questions. Tout au moins éclaire-t-elle sur le registre de ces interventions générées par la prise en compte de la fonction du répondant. J'y reviendrai ultérieurement. Là encore, c'est la question des conditions de cet enclenchement ou de la poursuite de la dynamique symbolique qui est posée et - on le sait aujourd'hui des nécessaires réaménagements du cadre susceptibles d'alimenter une rencontre aux vertus symboligènes. Dès lors, la question du cadre et celle du type de transfert qui y est engagé sont centrales. De telles évolutions à l'égard du cadre classique se fondent sur un dispositif qui associe l'expérience d'une compatibilité psychique avec l'autre, tout en représentant le miroir du négatif de ce que le patient ne parvient pas encore à exprimer (Roussillon, 2013). La prédisposition à cette double fonction enchâssée nous engage à poursuivre la réflexion sur la modalité d'un transfert adapté à cette forme de clinique et aux aménagements qu'elle nécessite.

\section{Cadre et transfert dans les problématiques narcissiques-identitaires}

Ce n'est certainement pas le fruit du hasard si cette réflexion sur les aménagements du cadre fut bien souvent menée par des cliniciens se frottant au processus adolescent et à ses impasses. Une certaine clinique du pubertaire qui s'impose par l'intensité des enjeux narcissiques et objectaux qu'elle révèle au fur et à mesure de l'accompagnement psychothérapique. Ainsi Jean-Luc Donnet a-t-il indiqué en ces termes la problématique à considérer au plus fort d'une rencontre dont l'un des objectifs se caractérise par le circuit d'échanges qu'elle est en mesure ou non d'établir: «Comment pourrons-nous être prêts à faire "feu de tout bois", disponibles pour toute intervention concernant l'aménagement optimal du cadre, si nous sommes 
trop profondément pénétrés de ce que seule l'interprétation de transfert aurait une valeur authentiquement restitutive?» (Donnet, 1983, p. 46-47). En s'appuyant sur cette réflexion, d'aucuns ont soutenu la notion de transfert sur la séance, en tant qu'elle recentre la dynamique de la séance sur l'appropriation subjective des éléments qui la composent: le transfert sur la séance devient l'expression de ce qui doit être enfin qualifié du fait de la rencontre. L'enjeu serait d'assurer une "affectation»-au sens de réception de l'adresse par arrimage de l'affect chez l'analyste - à ce qui s'offre au décours de la séance. On assiste dès lors à un travail préalable à tout principe de secondarisation du matériel psychique mis à disposition, par lequel il s'agit de poser les bases d'une transformation psychique de l'expérience à travers l'autre. Ainsi l'enjeu de la séance, dans de telles problématiques, ne s'inscrit-il pas tant dans l'analyse de l'événement remémoré que dans sa qualification, dans une inscription qui ne pourra se réaliser pour le sujet qu'en ayant préalablement effectué un certain trajet à travers l'autre, ce qui lui permettra d'assurer un certain arrimage de l'élément psychique incriminé. Tout l'enjeu est alors d'assigner une preuve d'existence de l'événement jadis traversé, d'inscrire l'évidence qu'il s'est passé quelque chose de l'ordre de «l'impressionnable». L'inscription psychique de l'expérience n'est possible qu'en «impressionnant» un autre capable, par l'usage d'une surface sensible et malléable interne, de donner sens à ce qui restait en suspens, indéfini, et de ce fait, inassimilable:

Je viens de l'évoquer rapidement, le transfert peut n'être considéré que comme la réédition d'une relation passée se reproduisant avec le praticien au sein de la rencontre clinique. Mais il peut aussi être considéré comme une tentative pour faire advenir ce qui n'a pu avoir lieu dans les situations plus ou moins traumatiques de l'histoire, comme D. W. Winnicott l'a fortement souligné. (Roussillon, 2013, p. 33).

Nous insistons avec d'autres sur l'importance de ne pas exercer, dans ce type de situations cliniques, l'analyse du transfert exclusivement sur ce qui se joue avec l'analyste mais dans quelque chose qui s'apparenterait plus à un transfert sur la séance, c'est-à-dire en cherchant à affecter - au double sens du terme - ce qui se réalise enfin, en cours de séance comme à l'extérieur, dans toute rencontre impliquant un répondant digne de ce nom. C'est, là encore, tout le mérite de cliniciens de l'adolescence que d'avoir insisté sur l'importance d'un «espace psychique élargi» (Jeammet, 1980) et de son 
influence dans les aménagements du dispositif thérapeutique. Il s'agit tout à la fois de reconnaître la charge émotionnelle du matériau exondé et de lui accorder un statut afin qu'il accède au rang de matériel psychique exploitable dans le processus psychothérapique, eu égard aux atteintes narcissiques et objectales massives dont le patient fait l'objet. En d'autres termes, ce sont les modalités de réception de l'adresse qui sont au centre du dispositif psychothérapique. Il en va d'une certaine qualité de présence dans la rencontre par laquelle quelque chose est ressenti ensemble, tout en permettant un certain désajustement face à l'expérience, modalité indispensable pour que l'incompréhensible prenne enfin sens.

Pour mesurer l'importance de ce processus, il faut convoquer ce que G. Haag énonce au sujet du "point de rebond», ce processus de transformation que l'environnement réalise auprès du nourrisson en qualifiant ce qui était inqualifiable pour l'infans. Elle insiste alors sur l'empathie suffisante mais aussi sur un certain décalage dans la réception de l'adresse. Ce sont ces effets conjugués qui garantiraient le prolongement du processus de transformation de l'expérience, initié par l'adulte, par l'enfant lui-même:

Cette hypothèse du point de retour avec suffisamment de mêmeté, de trouvailles d'empathie, de moments fusionnels dans l'émotion envoyée vers l'autre mais aussi suffisamment de désajustement, je crois que cela rejoint le terme bionien de transformation. La mère qui répond sur un autre mode, qui va proposer une forme vocale ou kinesthésique modulant une intensité d'angoisse ou d'extase, c'est cela qui ferait le point de rebond. (Haag, 2004, p. 31-32).

Ce jeu, où se manifeste suffisamment de semblable mais où le différent est également assuré, conditionne la possible appropriation par le sujet d'une fonction de transformation jusqu'alors détenue par l'autre. C'est bien cette question du relais qui est déterminante dans l'établissement d'une œuvre de symbolisation. Sans la mise en place de ce circuit de pensée à l'orée de la vie psychique du sujet, où l'expérience peut être partagée tout en s'appuyant sur l'assurance d'une singularité irréductible dans la rencontre avec l'autre, il est à craindre que l'œuvre de symbolisation ne soit entravée pour le sujet du fait de l'insistance d'un registre de l'ordre de l'impensable, c'est-à-dire ce qui reste par définition non transformable. Face à de telles carences dans les conditions d'accueil de l'environnement primordial, la gageure dans le cadre d'un accompagnement psychothérapique est majeure: elle se confond 
avec le risque, pour certains patients, de remettre sa vie psychique entre les mains d'un seul. Comment accepter une seconde fois de rencontrer la faillibilité de l'autre? La résurgence de situations intolérables est annoncée et n'est pas Winnicott qui veut... C'est ici que le dispositif institutionnel est convoqué, en tant qu'il est constitué de cadres associés dont la complémentarité assurerait tout à la fois la nécessaire diffraction du transfert dans des situations à la limite de la psychose et une condition indispensable pour le maintien du «cadre de l'analyste, lequel ne doit être ni ambigu, ni fluctuant, ni altéré» (Bleger, 1979, p. 275).

\section{La fonction du répondant en institution}

En quoi, dès lors, l'institution dont l'identité est sans conteste psychanalytique, peut-elle apporter une valeur ajoutée auprès de patients présentant de telles problématiques identitaires-narcissiques? Il ne s'agit pas de faire ici la liste exhaustive des fonctions inhérentes à tout dispositif institutionnel. Je souhaite principalement insister sur le concours qu'il permet dans l'établissement d'une matrice de contact, c'est-à-dire d'un dispositif sensible, capable de recevoir, d'inscrire et de formuler l'impact de l'expérience en présence d'autrui.

On se souvient de ce que Freud énonce au sujet de la dynamique de représentations en s'appuyant sur l'exemple du bloc-notes magique: il s'agit pour l'appareil psychique d'assurer tout à la fois la durabilité des traces psychiques dans le système mnémonique et l'effacement régulier des excitations provoquées de façon continue. Freud insiste sur la discontinuité de ce processus à l'origine, selon lui, «de l'apparition de la représentation du temps » (Freud, 1925, p. 143). Il est certain que l'un des enjeux principaux dans le travail psychothérapique de patients relevant de troubles narcissiques et identitaires graves correspond à cette possibilité recouvrée ou trouvée de se laisser aller à l'alternance de ces mouvements par lesquels excitations et perceptions se conjuguent pour former un système représentationnel, au rythme et selon un intervalle de temps que seul le sujet doit pouvoir définir comme ajustés. Ce rapport au temps, en tant qu'il assure le principe d'appropriation d'un matériel psychique jusqu'à présent inassimilable, est souvent tributaire d'un agencement de cadres que l'institution, par l'enchâssement de lieux, de moments, et par leur cohérence, est en mesure d'offrir en complémentarité avec le temps des séances.

Afin de préciser cette idée, il me semble pertinent de réaliser un rapprochement avec ce que R. Barthes avançait, dans le cadre de sa conférence 
inaugurale au Collège de France, sur la tension qui s'exerce, chez tout être humain, entre idiorythmie et hétérorythmie. La distinction s'établit dans un constat que Barthes expose dans une mise en scène de la vie quotidienne:

De ma fenêtre ( $1^{\text {er }}$ décembre 1976), je vois une mère tenant son gosse par la main et poussant la poussette vide devant elle. Elle allait imperturbablement à son pas, le gosse était tiré, cahoté, contraint à courir tout le temps, comme un animal ou une victime sadienne qu'on fouette. Elle va à son rythme, sans savoir que le rythme du gosse est autre. Et pourtant, c'est sa mère! Le pouvoir, - la subtilité du pouvoir - passe par la dysrythmie, l'hétérorythmie. (Barthes, 2002, p. 240)

L'hétérorythmie s'impose sur tout sujet comme la contrainte sociale qu'une société a établi pour tous ses membres. Elle se caractérise par un certain rapport au temps que normes et convenances ont fixé dans la durée. A contrario, chaque sujet entretient un commerce avec le temps vécu - l'idiorythmie -, par lequel il s'offre le loisir de pouvoir désirer, penser, rêver et fantasmer au rythme qui est le sien et malgré les contraintes exercées par le monde externe. Ce contexte institue, selon Barthes, un «tiraillement» sur lequel se construit la trame existentielle de tout un chacun. C'est un fait: certains patients interrogent plus que d'autres la manière dont les institutions de soin tentent de faire coexister hétérorythmie et idiorythmie au sein de leur organisation. Cette sollicitation, si l'on se réfère à ce qui a été évoqué plus haut au sujet de l'inscription de la représentation dans l'appareil psychique, s'explique par la correspondance qu'il convient d'établir entre dynamique de la pensée et constitution d'un rapport au temps, du fait de la discontinuité à laquelle le système représentationnel est soumis. Dès lors, on peut considérer qu'au plus fort de la remobilisation de la symbolisation chez le patient, c'est ce rapport au temps, représenté par l'organisation choisie par une institution, qui va être interrogée et, bien plus, contestée voire attaquée $^{3}$. On peut penser que les carences plus ou moins importantes dans l'établissement d'une symbolisation primaire aux tout premiers temps de la rencontre avec l'autre ont connu un contexte de dysrythmie tout particulièrement menaçant. L'émergence d'une pensée propre, s'appuyant sur l'expérience d'une idiorythmie tolérée et, bien plus, reconnue par l'autre, s'accompagne souvent de réactions agressives à l'égard de tout rappel des impondérables occasionnés par les contraintes hétérorythmiques. La clinique de l'adolescence, là encore, s'impose en un modèle indépassable. Il 
devient alors particulièrement précieux que l'institution se réserve ce registre afin de préserver la découverte d'un rythme propre au sein de la thérapie.

Cette réalité clinique, commune dans l'accompagnement psychothérapique de sujets soumis à des problématiques narcissiques et identitaires, renvoie à la fonction de répondant et à la manière dont elle est susceptible de s'exercer dans le cadre thérapeutique. C'est là qu'il faut, à mon sens, insister une dernière fois sur le rôle de l'institution dans le déploiement des effets de symbolisation. La question devient la suivante: en quoi le fait institutionnel est-il un outil déterminant pour travailler cette certitude d'un dispositif contenant à la hauteur du défi engagé chez des sujets souffrant d'une défaillance fondamentale du répondant? Toute structure de soin est mise en demeure de préciser les axes directeurs de cette fonction de répondant, en tant qu'ils définissent une certaine modalité de vivre avec - avec l'autre, le semblable et le différent, mais aussi avec l'autre en soi et les modifications que ce vivre avec engendre jour après jour. Cette question de vivre avec devient d'autant plus cruciale qu'elle rencontre le risque d'une confusion à mesure que les conditions de symbolisation primaire sont révélées, pour le patient, dans sa rencontre avec le thérapeute. L'enjeu s'impose alors dans la proposition suivante: vivre avec sans se perdre dans. Enjeu essentiel pour des sujets aux problématiques que l'on a coutume de nommer «identitaires-narcissiques».

Comment l'institution formée par une approche résolument psychanalytique peut-elle se prêter à entretenir l'espoir - une utopie, sans nul doute - d'une coexistence harmonieuse entre l'idiorythmie à constituer, au rythme d'une pensée propre, singulière, et indispensable pour pouvoir jouer, rêver, imaginer, créer et penser par soi-même, et cette hétérorythmie imposée par le social, avec ses convenances, ses coutumes et ses lois, mais nécessaire pour assurer les enjeux d'un vivre avec? Il me semble que l'institution d'orientation psychanalytique est fondamentalement engagée dans cette recherche de coexistence, et que cette dynamique, imparfaite mais toujours relancée, est vitale pour des sujets aux prises avec des problématiques narcissique-identitaires.

Vincent Cornalba vincentcornalba@free.fr

\section{Notes}

1. Un enjeu que suggère, par exemple, la situation clinique de Nour, présentée par Jérôme Ballif dans ce numéro. 
2. Ici encore, l'accompagnement clinique de Nour, présenté dans la contribution de J. Ballif, l'illustre avec force. J'en veux pour preuve supplémentaire ce qui se joue, par exemple, auprès d'adolescents se scarifiant: il s'agit tout autant de tenter de gérer la souffrance psychique à travers des stratégies auto-calmantes, où le sujet parvient a minima à maîtriser l'intensité de la douleur ressentie, qu'à vérifier l'effet qu'une telle atteinte sur le corps propre produit chez autrui. Force est de constater que l'adolescent scarificateur s'arrange très souvent pour que son acte soit découvert, quitte à transposer ce besoin sur un mode fortuit: le hasard de la découverte est la plupart du temps provoqué...

3. J'ai à l'esprit ces fameuses situations d'urgence qui s'invitent violemment dans les réunions cliniques et/ou institutionnelles où la recherche d'un autre temps, plus conforme à la possibilité de penser, d'associer sur les situations cliniques, de rêver sur les patients, tente de résister aux seules contraintes logistiques et organisationnelles.

\section{Références}

Avron, O. (2010). Psychodrame: la technique du double, Dialogue, 189, 95-108.

Barthes, R. (2002). Comment vivre ensemble. Cours et séminaires au Collège de France. Paris: Éditions du Seuil, 1976-1977.

Bleger, J. (1979). Psychanalyse du cadre psychanalytique. Dans R. Kaës (dir.). Crise, Rupture et dépassement (p. 257-276). Paris: Dunod.

Donnet, J.-L. (1983). Sur la rencontre avec l'adolescent, Adolescence, 1, 45-61.

Freud, S. (1925). Note sur le «Bloc magique». Dans Euvres complètes - Psychanalyse, XVII (p. 137-143). Paris: Presses universitaires de France.

Freud, S. (1913). Le début du traitement. Dans La technique psychanalytique (p. 80-104). Paris: Presses universitaires de France.

Freud, S. (1895). Esquisse d'une psychologie scientifique. Dans La naissance de la psychanalyse (p. 307-396). Paris: Presses universitaires de France, 1991.

Gutton, P. (2013). Réflexions sur le statut de l'analyste dans la cure des adolescents. Cahiers de psychologie clinique, 1 (40), 165-180.

Haag, G. (2004). Sexualité orale et Moi corporel. Topique, 87, 23-45.

Jeammet, P. (1980). Réalité externe et réalité interne. Importance et spécificité de leur articulation à l'adolescence. Revue française de psychanalyse, 44 (3-4), 481-521.

Pinel, J.-P. (2011). Les adolescents en grandes difficultés psychosociales: errance subjective et délogement généalogique, Connexions, 2 (96), 9-26.

Roussillon, R. (2013). Diversité et complexité des pratiques cliniques. Cahiers de psychologie clinique, 1 (40), 29-45.

Winnicott, D. W. (1975). La crainte de l'effondrement. Dans La crainte de l'effondrement et autres situations cliniques (p. 205-216). Paris: Gallimard, 2000. 